\title{
SOME ANALYTICAL PROPERTIES OF CONTINUOUS STATIONARY MARKOV TRANSITION FUNCTIONS $\left({ }^{(}\right)$
}

\author{
BY \\ DAVID G. KENDALL
}

Summary. A systematic treatment of Markov processes with Euclidean state-spaces has recently been presented by Doob [1], the restriction on the nature of the state-space being associated with the very illuminating probabilistic method which he uses throughout. At about the same time a new step was taken by Kolmogorov [4] who established for countable statespaces the existence and finiteness of the derivative of the transition-function $p_{i j}(t)$ at $t=0+$ when $i \neq j$. In this paper some of Doob's and Kolmogorov's results are combined and shown to be valid (when suitably formulated) for an arbitrary state-space. For the sake of a generality which proves useful in the discussion of existence theorems the transition-function $P_{t}(x, A)$ is not assumed to be "honest"; i.e., if $X$ is the state-space then it is supposed that $P_{t}(x, X) \leqq 1$.

1. Measurability in the state-space. Let $x$ be a general point in a nonvacuous set $X$; in the probabilistic applications $x$ is a possible state of a system and so $X$ will be called the state-space. We shall need the concept "measurable subset of $X$ " and we suppose these subsets to constitute a Borel field $\mathcal{F}$; let $\mathcal{F}^{2}$ be the smallest Borel field of subsets of $X^{2}(=X \times X)$ with regard to which the two "coordinate-functions" on $X^{2}$ are measurable $\left({ }^{2}\right)$. Let $D$ denote the "diagonal" in $X^{2}$ (i.e., the graph of the identical map from $X$ to itself). We shall throughout this paper adopt

Assumption A. $D \in \mathcal{F}^{2}$.

As an immediate consequence of A (because all "sections" of $\mathfrak{F}^{2}$-sets are $\mathcal{f}$-sets) we have:

THEOREM 1.1. All one-point sets belong to $\mathcal{F}$.

In Feller [2] the truth of Theorem 1.1 is a requirement (the only one) imposed on $\mathcal{F}$, but we need the stronger assumption $\mathrm{A}$ in the proof of Theorem 3.4.

It is a little unsatisfactory that $\mathrm{A}$ should refer to $\left(X^{2}, \mathcal{F}^{2}\right)$ rather than to $(X, \mathcal{F})$, and so it is of interest that the following is sufficient for the truth of A:

Received by the editors May 9, 1953 and, in revised form, May 25, 1954.

(1) Work done partly under the sponsorship of the U. S. Office of Naval Research.

(2) Thus $\mathcal{F}^{2}$ is the Borel field generated by the subsets of $X^{2}$ of the form $A \times B$, where $A$ and $B$ are in $\mathcal{F}$. 
Assumption $A_{0}$. There is a countable class $\mathcal{E}$ of $\mathcal{F}$-sets, the elements of which separate the points of $X$.

(We say that "the elements of $\varepsilon$ separate the points of $X$ " when, $x$ and $y$ being arbitrary distinct points of $X$, there exist disjoint $\varepsilon_{\text {-sets }} E_{x y}^{(1)}$ and $E_{x y}^{(2)}$ containing $x$ and $y$ respectively.) The proof that $\mathrm{A}_{0}$ implies $\mathrm{A}$ consists in noting that when $\mathrm{A}_{0}$ is true then the complement of $D$ can be expressed as a countable union of sets of the form $E^{(1)} \times E^{(2)}$ where $E^{(1)} \in \mathcal{E}$ and $E^{(2)} \in \mathcal{E}$.

$\mathrm{A}_{0}$ (and so also $\mathrm{A}$ ) is satisfied in all the usual applications. This is most easily seen by observing that $A_{0}$ is equivalent to:

Assumption $A_{1}$. The Borel field $\mathcal{F}$ contains all the open sets of a Hausdorff topology for $X$ satisfying the second axiom of countability.

(In practice $\mathcal{F}$ will usually be the smallest Borel field containing all the open sets of such a topology.)

Proof that $A_{0} \equiv A_{1}$. If $A_{0}$ is true we take $\varepsilon$ as the sub-basis of a topology. If $A_{1}$ is true we take any countable basis of the topology as the class $\varepsilon$.

It is to be noted that if we were to adopt $A_{0}\left(\equiv A_{1}\right)$ in preference to $A$ then we should be committed to the assumption that the state-space $X$ is at most of the power of the continuum.

2. Axioms to be satisfied by the transition-functions. Let $T$ be the nonnegative part of the real line. We shall be concerned with transition-functions $P .(\cdot, \cdot)$ which map $T \times X \times \mathcal{F}$ into the closed interval $[0,1]$ and which satisfy the following axioms. and

$$
P_{t}(x, X) \leqq 1 .
$$

$\mathrm{P}_{2}$. For each $t \in T$ and $A \in \mathcal{F}, P_{t}(\cdot, A)$ is $\mathcal{F}$-measurable.

$\mathrm{P}_{3}$. For each $u$ and $v$ in $T$, for each $x \in X$ and $A \in \mathcal{F}$,

$$
P_{u+v}(x, A)=\int P_{v}(\cdot, A) d P_{u}(x, \cdot) .
$$

$\mathrm{P}_{4} \cdot P_{0}(\cdot, \cdot)=\Delta(\cdot, \cdot)$.

$\mathrm{P}_{5}$. For each $x \in X$,

$$
\lim _{t \rightarrow 0+} P_{t}(x,\{x\})=1 .
$$

The function $\Delta$ referred to in $\mathrm{P}_{4}$ is defined as follows:

$$
\Delta(x, A)= \begin{cases}1 & \text { if } x \in A, \\ 0 & \text { if } x \notin A .\end{cases}
$$

If the sign of inequality in (1) were replaced by the sign of equality, the assumptions $P_{1}$ to $P_{b}$ would define a set of stationary Markov transition functions of the "jumping" type (the "jumping" behaviour is implied by $P_{8}$ ). 
Even in the probabilistic applications, however, it is of interest that none of the theorems to be proved here requires the sign of equality in (1).

If $X$ were the real line, if $\mathcal{F}$ were the class of Borel sets, and if equality were required in (1), then we could set up a probability-measure on functionspace and employ the probabilistic arguments used by Doob in Chapter VI of his book [1], but such methods are not available in the general situation considered here. Our present point of view is more nearly that of Feller [2], although Feller imposed on the $P$-functions a set of conditions much stronger than $\mathrm{P}_{1}$ to $\mathrm{P}_{5}$.

3. Some immediate consequences of the axioms.

Theorem 3.1. For each $x \in X$, the function $P .(x,\{x\})$ is "super-multiplicativen on $T$.

Proof.

$$
P_{u+v}(x,\{x\}) \geqq \int_{\{x\}} P_{v}(\cdot,\{x\}) d P_{u}(x, \cdot)=P_{u}(x,\{x\}) P_{v}(x,\{x\}) .
$$

TheOREM 3.2. For each $x \in X$, the function $P .(x,\{x\})$ is positive on $T$.

Proof.

$$
P_{t}(x,\{x\}) \geqq\left[P_{t / n}(x,\{x\})\right]^{n} \quad(n \geqq 1),
$$

and the right-hand side is positive for all sufficiently large $n$ (in virtue of $\mathrm{P}_{6}$ ).

Theorem 3.3. If $u \in T, v \in T, x \in X$ and $A \in \mathcal{F}$ then

$$
\left|P_{u}(x, A)-P_{v}(x, A)\right| \leqq 1-P_{|u-v|}(x,\{x\}),
$$

and so for each fixed $x \in X, P .(x, A)$ is uniformly continuous on $T$ (righthandedly at $t=0$ ) uniformly with regard to $A \in \mathcal{F}$.

For let $u=v+t$ where $t \geqq 0$. Then

$$
P_{u}(x, A)-P_{v}(x, A)=\int_{X-\{x\}} P_{v}(\cdot, A) d P_{t}(x, \cdot)-P_{v}(x, A)\left[1-P_{t}(x,\{x\})\right] .
$$

Thus, taking the second term on the right-hand side,

$$
P_{u}(x, A)-P_{v}(x, A) \geqq-\left[1-P_{t}(x,\{x\})\right],
$$

and taking the first term on the right-hand side,

$$
P_{u}(x, A)-P_{v}(x, A) \leqq P_{t}(x, X-\{x\}) \leqq 1-P_{t}(x,\{x\}) .
$$

The last clause of Theorem 3.3 (which now follows at once) answers a question raised by Doob [1, p. 257].

ThEOREM 3.4. For each $t \in T$ the real-valued function $g$ defined over $X$ by 


$$
g(x) \equiv P_{t}(x,\{x\})
$$

is $\mathcal{F}$-measurable. write

(It is here that we need Assumption A.) If $E \subseteq X^{2}$ and $x \in X$, we shall

$$
E_{x} \equiv\{y:(x, y) \in E\} .
$$

Then if $E \in \mathcal{F}^{2}$ we shall have $E_{x} \in \mathcal{F}$, and so for each $t \in T$ we can define a function $h$ over $X$ by

$$
h(x) \equiv P_{t}\left(x, E_{x}\right) .
$$

We shall prove that $h$ is $\mathcal{F}$-measurable; the required result will then follow on putting $E=D$.

Suppose first that $E=A \times B$, where $A \in \mathcal{F}$ and $B \in \mathcal{F}$. Then

$$
h(x)=\Delta(x, A) P_{t}(x, B)
$$

and each factor is $\mathcal{F}$-measurable. Let $G$ be the class of $\mathcal{F}^{2}$-sets $E$ such that $h$ is $\mathcal{F}$-measurable. Then if $R$ is the field of finite disjoint unions of measurable rectangles $A \times B$, we have $R \subseteq G \subseteq \mathcal{F}^{2}$. But $G$ is a monotone $\operatorname{class}\left({ }^{3}\right)$ and $\mathcal{F}^{2}$ is the smallest Borel field covering $R$, so $G=\mathcal{F}^{2}$.

4. The function $q$.

THEOREM 4.1. The limit

$$
q(x) \equiv \lim _{t \rightarrow 0+} \frac{1-P_{t}(x,\{x\})}{t}
$$

exists for all $x \in X$ (but may be equal to $+\infty$ ). The function $q$ is $\mathcal{F}$-measurable, and for all $t \in T$ and $x \in X$ we have

$$
1-P_{t}(x,\{x\}) \leqq 1-e^{-q(x) t} .
$$

It will be noted that (3) and (4) together give

$$
\left|P_{u}(x, A)-P_{v}(x, A)\right| \leqq 1-e^{-|u-v| q(x)} .
$$

The $\mathcal{F}$-measurability of $q$ (once it is shown to exist) is an immediate consequence of Theorem 3.4. The fact that $q(x)$ can actually assume the value $+\infty$ (even when $X$ is countable and the sign of equality holds in (1)) is shown by an example given by Kolmogorov [4]. The existence of the limit for Euclidean $(X, \mathscr{F})$ was proved by Doob (assuming the sign of equality to hold in (1)) [1, p. 258], but his methods are not available here.

$\left(^{3}\right)$ For the definition of a monotone class of sets see P. R. Halmos, Measure theory, New York, 1950. (Halmos writes "algebra" and " $\sigma$-algebra" where we write "field" and "Borel field.") 
We shall show that the existence of the limit follows from a simple lemma about sub-additive functions (for this, see Hille [3, p. 143]). For a fixed $x \in X$ let $f(t) \equiv-\log P_{t}(x,\{x\})$. Then$$
0 \leqq f(t)<\infty
$$$$
(0<t<\infty) \text {, }
$$
(b) $f(u+v) \leqq f(u)+f(v)$ $(0<u, v<\infty)$,
(c) $f(0+)=0$,

and so $f(t) / t$ has a limit as $t \rightarrow 0$ and

$$
0 \leqq \lim _{t \rightarrow 0+} \frac{f(t)}{t}=\sup _{t>0} \frac{f(t)}{t} \leqq \infty .
$$

Write $q(x)$ for the common value of the limit and the supremum. Then $f(t) \leqq q(x) t(t \geqq 0)$ (this gives (4)), and so when $q(x)=0$ it will follow that $P_{t}(x,\{x\})=1$ for all $t$, the existence of the limit then being trivial. When $q(x)>0$ then $f(t)$ will be positive for all sufficiently small nonzero values of $t$ and we can then write

$$
\frac{1-P_{t}(x,\{x\})}{t}=\frac{1-e^{-f(t)}}{f(t)} \frac{f(t)}{t} \rightarrow q(x) .
$$

This completes the proof of Theorem 4.1.

It is usual to call $x$, an absorbing state when $q(x)=0$, and Lévy [5; 6] calls $x$ an instantaneous state when $q(x)=\infty$.

5. The behaviour of $P_{t}(x, A) / t$ when $t \rightarrow 0$. We assumed in $\mathrm{P}_{5}$ that

$$
\lim _{t \rightarrow 0+} P_{t}(x,\{x\})=1
$$

for each $x \in X$; for some sets $A \in \mathcal{F}$ (e.g., for all finite sets) it will be true that the limit in (6) exists uniformly for all $x \in A$.

We shall denote the class of such $\mathcal{F}$-sets by $R_{u}$, and we then have:

TheOREM 5.1. $\mathbb{R}_{u}$ is a ring of $\mathcal{F}$-sets, and each $\mathcal{F}$-measurable subset of an $R_{u}$-set is also in $R_{u}$.

Only exceptionally will the whole state-space $X \in R_{u}$ (for example, this happens if $X$ is finite) and so $R_{u}$ will not in general be a field of sets.

Following Feller [2] and Doob [1] we shall say that a set $A$ is $q$-bounded if $A \in \mathcal{F}$ and if $\{q(x): x \in A\}$ is an empty or bounded set of real numbers. We shall write $A \in R_{0}$ if and only if there exists an integer $N \geqq 0$ such that

$$
A=B \cup\left\{x_{1}, x_{2}, \cdots, x_{N}\right\}
$$

where $B$ is $q$-bounded and each $x_{i}$ is an instantaneous state $(1 \leqq i \leqq N)$. We then have: 
THEOREM 5.2. $R_{0}$ is a sub-ring of $\mathbb{R}_{u}$, and every $\mathcal{F}$-measurable subset of an $R_{0}$-set is also in $R_{0}$.

This is an immediate consequence of (4). [The use in the following arguments of the larger ring $R_{u}$ instead of $R_{0}$ was suggested by J. L. Doob.]

We shall now prove

Theorem 5.3. If $A \in R_{u}$ and $x \notin A$ then the limit

$$
q(x, A) \equiv \lim _{t \rightarrow 0+} \frac{P_{t}(x, A) !}{t}
$$

exists and is finite.

This result is more general than one of Doob [1, p. 261] in that we do not require $q(x)$ to be finite. It is a generalisation of a theorem of Kolmogorov [4] in which (i) $X$ is countable, (ii) $A$ is a one-point set, and (iii) equality is supposed to hold in (1). The proof is based on Kolmogorov's proof of his theorem; he begins by proving an interesting preliminary result of which the following is the appropriate generalisation.

THEOREM 5.4. Let $R$ be a fixed element of $R_{u}$ and let $\epsilon$ be a fixed positive number less than 1/4. Let $\tau$ be a positive number (depending on $R$ and $\epsilon$ ) such that

$$
1-P_{t}(y,\{y\})<\epsilon
$$

whenever $y \in R$ and $0 \leqq t \leqq \tau$. Then if $A$ is any $\mathcal{F}$-measurable subset of $R$ and if $x \in R-A$, we shall have

$$
(1-4 \epsilon) \frac{P_{u}(x, A)}{u} \leqq \frac{P_{v}(x, A)}{v}
$$

provided that $0<v \leqq \tau$ and $0<\dot{u} / v \leqq \epsilon$.

Let $A$ be a particular $\mathcal{F}$-measurable subset of $R$ and $x$ a particular element of $R-A$. Let $u$ and $v$ satisfy the stated inequalities and let $n \equiv[v / u] \geqq 4$ be the integer part of $v / u$. We define $F_{m}(y, B)$ for all $y \in X, B \in \mathcal{F}$ and $m \geqq 1$ recurrently as follows:

$$
\begin{aligned}
F_{1}(y, B) & \equiv P_{u}(y, B), \\
F_{m+1}(y, B) & \equiv \int_{X-A} P_{u}(\cdot, B) d F_{m}(y, \cdot)
\end{aligned}
$$

(it will be noted that $A$ enters into the definition of the $F$-functions). (For each $m$ and $y, F_{m}(y, \cdot)$ is a totally-finite measure on $(X, \mathcal{F})$, and the reader may find it helpful in a first reading of the following argument to ignore (1) and to think of $F_{m}(y, B)$ as the probability of reaching some state in $B$ from the initial state $y$ in time $m u$, the set of states $A$ being avoided at the epochs 
$u, 2 u, \cdots,(m-1) u$. But the argument is analytic and not probabilistic, unless (1) is modified by an obvious re-norming.) By induction on $m$ we obtain the identity

$$
P_{t}(x, B)=\sum_{j=1}^{m-1} \int_{A} P_{t-j u}(\cdot, B) d F_{j}(x, \cdot)+\int P_{t-m u}(\cdot, B) d F_{m}(x, \cdot),
$$

where $B \in \mathcal{F}, m \geqq 1$ and $t \geqq m u$. (The first terms on the right-hand side are absent when $m=1$.) $\left({ }^{4}\right)$ Following Kolmogorov we now apply (10) three times.

(i) Put $B=A, m=n, t=v$. Observing that $A \subseteq X-\{x\}$ and that $x \in R$, we have $P_{v}(x, A) \leqq 1-P_{v}(x,\{x\})<\epsilon$, and also

$$
\begin{aligned}
P_{v}(x, A) & \geqq \sum_{j=1}^{n} \int_{A} P_{v-j u}(\cdot, A) d F_{j}(x, \cdot) \\
& \geqq \sum_{j=1}^{n} \int_{A} P_{v-j u}(y,\{y\}) F_{j}(x, d y) \\
& \geqq(1-\epsilon) \sum_{j=1}^{n} F_{j}(x, A),
\end{aligned}
$$

so that

$$
\sum_{j=1}^{n} F_{j}(x, A)<\epsilon /(1-\epsilon) .
$$

(ii) Next put $B=\{x\}$ and $t=m u$, where $1 \leqq m \leqq n-1$. Then we get

$$
P_{m u}(x,\{x\}) \leqq \sum_{j=1}^{m-1} F_{j}(x, A)+F_{m}(x,\{x\}),
$$

and so if $1 \leqq m \leqq n-1$,

$$
F_{m}(x,\{x\})>P_{m u}(x,\{x\})-\epsilon /(1-\epsilon)>(1-3 \epsilon) /(1-\epsilon) .
$$

(iii) As in (i) let $B=A, m=n$, and $t=v$, but now note that

$$
F_{m+1}(x, C) \geqq P_{u}(x, C) F_{m}(x,\{x\})
$$

for all $C \in \mathcal{F}$ and $m \geqq 1$. It then follows that

$$
\begin{aligned}
P_{v}(x, A) & \geqq \int_{A} P_{v-u}(\cdot, A) d P_{u}(x, \cdot)+\sum_{j=2}^{n} F_{j-1}(x,\{x\}) \int_{A} P_{v-j u}(\cdot, A) d P_{u}(x, \cdot) \\
& \geqq(1-\epsilon) P_{u}(x, A)+(n-1) \frac{1-3 \epsilon}{1-\epsilon}(1-\epsilon) P_{u}(x, A),
\end{aligned}
$$

(4) In proving this identity the inversions of the order of integration are to be justified by a lemma given by Feller [2, pp. 493-494], and not by Fubini's theorem. 
and so

$$
\frac{P_{v}(x, A)}{v} \geqq(1-3 \epsilon) \frac{n u}{v} \frac{P_{u}(x, A)}{u} .
$$

This last inequality, when combined with

$$
\frac{n u}{v} \geqq 1-\frac{u}{v} \geqq 1-\epsilon,
$$

leads at once to the required result.

We can now prove Theorem 5.3. Put $R \equiv A \cup\{x\} \in R_{u}$ and let $\epsilon<1 / 4$. Then from (8) (on letting $u \rightarrow 0$ ) we get

$$
(1-4 \epsilon) \limsup _{t \rightarrow 0+} P_{t}(x, A) / t \leqq P_{v}(x, A) / v
$$

provided that $0<v \leqq \tau$, and on letting $v \rightarrow 0$ we get

$$
(1-4 \epsilon) \limsup _{t \rightarrow 0+} P_{t}(x, A) / t \leqq \lim _{t \rightarrow 0+} P_{t}(x, A) / t \text {. }
$$

Equation (13) then shows that the limit superior is finite and (14) shows that it is equal to the limit inferior. This completes the proof of Theorem 5.3, but we can also prove the following additional result.

TheOREm 5.5. If $R \in R_{u}$ then there exist a positive $\tau$ and a finite $K$ (each depending on $R$ ) such that

$$
q(x, A) \leqq K P_{\tau}(x, A)
$$

whenever $A$ is an 7 -measurable subset of $R$ and $x \in R-A$.

Proof. Choose $\epsilon=1 / 8$, and then take $\tau=\tau(R, \epsilon)$ and $K=2 / \tau$ after letting $u \rightarrow 0$ in (8).

It is useful to extend the domain of definition of $q(x, \cdot)$ to the whole of $R_{u}$ by writing

$$
q(x, A) \equiv q(x, A-\{x\})
$$

for an arbitrary element $A$ of $R_{u}$. Once this is done we have

Theorem 5.6. For each $x \in X$ and $A \in R_{u}, q(x,\{x\})=0$ and $q(x, A) \leqq q(x)$.

We also have

TheOREM 5.7. For each $x \in X, q(x, \cdot)$ is a finite measure on $\left(X, \mathbb{R}_{u}\right)$, and for each $A \in \mathcal{R}_{u}, q(\cdot, A)$ is $\mathcal{F}$-measurable.

Proof. It is clear that $q(x, \cdot)$ is a finite weakly-additive measure on $\left(X, \mathrm{R}_{u}\right)$. Let then $\left\{A_{n}\right\}$ be a decreasing sequence of $\mathrm{R}_{u}$-sets having a void intersection; we must show that $q\left(x, A_{n}\right) \rightarrow 0$ as $n \rightarrow \infty$. 
Now we shall have $x \notin A_{n}$ if $n \geqq N$ (say), and if we identify $A_{N} \cup\{x\}$ with $R$ of Theorem 5.5 we get

$$
q\left(x, A_{n}\right) \leqq K P_{\tau}\left(x, A_{n}\right)
$$

where $K$ and $\tau$ do not depend on $n$. The result is now immediate. The $\mathcal{F}$-measurability of $q(\cdot, A)$ follows from the identity

$$
q(x, A)=\lim _{t \rightarrow 0+} \frac{P_{t}(x, A)-P_{t}(x,\{x\}) \Delta(x, A)}{t}
$$

and Theorem 3.4.

We close this section of the paper by noting the following consequence of Theorems 4.1 and 5.3.

Theorem 5.8. If $x \in X$ and $A \in \mathbb{R}_{u}$, then

$$
\left[\frac{d}{d t} P_{t}(x, A)\right]_{t=0+}=q(x, A)-q(x) \Delta(x, A) .
$$

6. The extension from $\left(X, \mathbb{R}_{u}\right)$ to $(X, \mathcal{F})$. So far $q(x, A)$ has not been defined unless $A \in R_{u}$. An additional assumption appears to be necessary at this point and (following a suggestion of Doob) we shall adopt

Assumption B. The state-space $X$ is the union of an increasing sequence of $R_{u}$-sets.

We note

Theorem 6.1. Assumption B is satisfied whenever the set I of instantaneous states is at most countably infinite.

(In particular B is satisfied if $I$ is void, and it is also satisfied if $X$ is countable.) The proof consists in observing that if the instantaneous states are $x_{1}, x_{2}, \cdots$ and if

$$
X_{r} \equiv\{x: q(x) \leqq r\} \cup\left\{x_{1}, x_{2}, \cdots, x_{r}\right\},
$$

then $X_{r} \in R_{u}$ and $X_{r} \uparrow X$.

Theorems for the proof of which B is required will be designated thus *. The first of these is

TheOREM 6.2.* $\mathcal{F}$ is the Borel field generated by $R_{u}$, and the unique extension $q(x, \cdot)$ of $q(x, \cdot)$ from $\left(X, R_{u}\right)$ to $(X, \mathcal{F})$ preserves the properties of $q(x, \cdot)$ stated in Theorems 5.6 and 5.7.

Proof. We have, if $A \in \mathcal{F}$,

$$
A=\bigcup_{r=1}^{\infty} A \cap X_{r}
$$

and 


$$
q(x, A)=\lim _{r \rightarrow \infty} q\left(x, A \cap X_{r}\right) .
$$

A theorem of Doob (which is easily generalised to the form appropriate here) gives a condition sufficient for the truth of (16) when $A$ is an $\mathcal{F}$-set and not necessarily an $R_{u}$-set. It is

Theorem 6.3.* Suppose that, for some fixed $x_{0} \in X$,

$$
\tilde{q}\left(x_{0}, X\right)=q\left(x_{0}\right)<\infty .
$$

Then

$$
\left[\frac{d}{d t} P_{t}\left(x_{0}, A\right)\right]_{t=0+}=\tilde{q}\left(x_{0}, A\right)-q\left(x_{0}\right) \Delta\left(x_{0}, A\right)
$$

for all $A \in \mathcal{F}$.

(In this connexion it is of interest to note that Kolmogorov [4] has constructed an example in which $X$ is countable, equality holds in (1), and $q\left(x_{0}, X\right)<q\left(x_{0}\right)<\infty$.)

Proof of Theorem 6.3.* (This is essentially Doob's own proof.) Let $\epsilon>0$ and $R \equiv X_{n}-\left\{x_{0}\right\} \in R_{u}$ where $n$ is to be chosen so large that

$$
q\left(x_{0}\right)-\epsilon<q\left(x_{0}, R\right) \leqq q\left(x_{0}\right) .
$$

It is enough to prove the required result when $x_{0} \notin A$ (the general case can then be dealt with very easily). Now if $A \in \mathcal{F}$ and $x_{0} \notin A$,

$$
\begin{aligned}
\left|P_{t}\left(x_{0}, A\right) / t-q\left(x_{0}, A\right)\right| \leqq & \left|P_{t}\left(x_{0}, A \cap R\right) / t-q\left(x_{0}, A \cap R\right)\right| \\
& +P_{t}\left(x_{0}, A-R\right) / t+q\left(x_{0}, A-R\right) .
\end{aligned}
$$

Of the three terms on the right-hand side the first will be less than $\epsilon$ for all sufficiently small values of $t$ because $A \cap R \in R_{u}$, and the third is at the most equal to

$$
\tilde{q}\left(x_{0}, X-R\right)=q\left(x_{0}\right)-q\left(x_{0}, R\right)<\epsilon .
$$

Also $A-R \subseteq\left(X-\left\{x_{0}\right\}\right)-R$ and $R \subseteq X-\left\{x_{0}\right\}$, and so for the remaining term we have the inequality

$$
P_{t}\left(x_{0}, A-R\right) / t \leqq \frac{1-P_{t}\left(x_{0},\left\{x_{0}\right\}\right)}{t}-\frac{P_{t}\left(x_{0}, R\right)}{t}
$$

and thus

$$
\limsup _{t \rightarrow 0+} P_{t}\left(x_{0}, A-R\right) / t \leqq q\left(x_{0}\right)-q\left(x_{0}, R\right)<\epsilon .
$$

It follows that

$$
\left|P_{t}\left(x_{0}, A\right) / t-q\left(x_{0}, A\right)\right|<3 \epsilon
$$


for all sufficiently small values of $t$, as was to be proved.

If there are no instantaneous states (so that Assumption B is automatically satisfied) and if $q(x, X)=q(x)$ for all $x \in X$, then we shall have

$$
\left[\frac{d}{d t} P_{t}(x, A)\right]_{t=0+}=q(x, A)-q(x) \Delta(x, A)
$$

for all $x \in X$ and all $A \in \mathcal{F}$. In these circumstances the arguments of Feller [2] become available, and it will follow that

$$
\frac{d}{d t} P_{t}(x, A)=-q(x) P_{t}(x, A)+\int P_{t}(\cdot, A) d \tilde{q}(x, \cdot)
$$

for all $t \geqq 0$, for all $x \in X$, and for all $A \in \mathcal{F}$. (This is the so-called "backward" equation.) The proof given by Feller [2, pp. 495-496] in the first instance establishes (19) only as an expression for the right-hand derivative, but both $P_{t}(x, A)$ and the right-hand side of (19) are continuous for $t \geqq 0$, and so $P_{t}(x, A)$ is differentiable for $t>0$.

7. The "dishonesty" function. We now abandon Assumption B and return to the general situation. The extent

$$
D_{t}(x) \equiv 1-P_{t}(x, X)
$$

to which $P_{t}(x, \cdot)$ falls short of being a probability measure may conveniently be called the dishonesty function, and for certain purposes the behaviour of the ratio $D_{t}(x) / t$ when $t \rightarrow 0$ is of interest. With the aid of Theorem 5.3 we can prove

ThEOREM 7.1. The limit

$$
\lim _{t \rightarrow 0+} \frac{D_{t}(x)}{t}
$$

exists and is finite for all $x \in X$.

Proof. Introduce a new state $\theta$ and put $\bar{X} \equiv X+\{\theta\}$, and let $\overline{\mathcal{F}}$ consist of all sets $\bar{A}$ of the forms $A$ and $A+\{\theta\}$ (where $A \in \mathcal{F}$ ). Assumption A will then be satisfied by the augmented pair $(\bar{X}, \overline{\mathcal{Y}})$ if it was satisfied originally. Now define

$$
\begin{array}{lr}
\bar{P}_{t}(\theta, \bar{A}) \equiv \Delta(\theta, \bar{A}) & (t \in T, \bar{A} \in \overline{\mathcal{F}}), \\
\bar{P}_{t}(x, A) \equiv P_{t}(x, A) & (t \in T, x \in X, A \in \mathcal{F}),
\end{array}
$$

and

$$
\bar{P}_{t}(x, A+\{\theta\}) \equiv P_{t}(x, A)+D_{t}(x)
$$

when $t \in T, x \in X$, and $A \in \mathcal{F}$. It will be found that the new system 
$\left\{\bar{P}_{t}(\bar{x}, \bar{A}): t \in T, \bar{x} \in \bar{X}, \bar{A} \in \overline{\mathcal{Y}}\right\}$ satisfies the axioms $\mathrm{P}_{1}$ to $\mathrm{P}_{5}$, and the required result now follows from Theorem 5.3 on putting $\bar{A} \equiv\{\theta\}$.

I am very much indebted to Professor J. L. Doob for a number of helpful suggestions in connexion with this work.

\section{REFERENCES}

1. J. L. Doob, Stochastic processes, New York, Wiley; London, Chapman and Hall, 1953.

2. W. Feller, On the integro-differential equations of purely discontinuous Markoff processes, Trans. Amer. Math. Soc. vol. 48 (1940) pp. 488-515. [See also corrigendum in vol. 58, p. 474].

3. E. Hille, Functional analysis and semi-groups, Amer. Math. Soc. Colloquium Publications, vol. 31, 1948.

4. A. N. Kolmogorov, On some problems concerning the differentiability of the transitionprobabilities in a temporally homogeneous Markov process having a denumerable set of states, Uzenye Zapiski, Moskovskogo Gosudarstvennogo Universiteta. Matematika (4) vol. 148 (1951) pp. 53-59.

5. P. Lévy, Systèmes markoviens et stationnaires: cas dénombrable, Ann. Ecole Norm. (3) vol. 68 (1951) pp. 327-381.

6. ——, Complément d l'étude des processus de Markoff, ibid. vol. 69 (1952) pp. 203-212.

Magdalen College, OXFORD, ENGLAND.

Princeton University, Princeton, N. J. 